\title{
Fabry Disease in Latin America: Data from the Fabry Registry
}

\author{
J. Villalobos • J.M. Politei • A.M. Martins • \\ G. Cabrera • H. Amartino • R. Lemay • S. Ospina • \\ S. Suarez Ordoñez $\cdot$ C. Varas
}

Received: 23 January 2012 /Revised: 07 June 2012 /Accepted: 12 June 2012 /Published online: 6 July 2012

(C) SSIEM and Springer-Verlag Berlin Heidelberg 2013

Communicated by: Olaf Bodamer

Competing interests: None declared

J. Villalobos

Department of Physiology, Central University of Venezuela, Luis

Razetti School of Medicine, Caracas, Venezuela

J.M. Politei

Neurology Service, Juan Fernandez Hospital, Buenos Aires,

Argentina

A.M. Martins

Centro de Referência em Erros Inatos do Metabolismo,

Universidade Federal de São Paulo, São Paulo, Brazil

G. Cabrera

Centro Médico del Viso, Buenos Aires, Argentina

H. Amartino

Child Neurology Department, Hospital Universitario Austral,

Buenos Aires, Argentina

R. Lemay

Genzyme, Cambridge, MA, USA

S. Ospina

Universidad del Rosario, Bogotá, Colombia

S. Suarez Ordoñez

Chiltern International, Buenos Aires, Argentina

C. Varas

Departamento Dermatología y ETS, Hospital San Pablo,

Coquimbo, Chile

J. Villalobos $(\bowtie)$

Cátedra de Fisiología, Universidad Central de Venezuela Instituto de Medicina Experimental, Piso 1 oficina 232 Ciudad

Universitaria. Avenida Los Ilustres,

Los Chaguaramos Caracas, Venezuela

e-mail: villazu@gmail.com

\begin{abstract}
The purpose of these analyses was to characterize demographic and baseline clinical characteristics of Latin American patients with Fabry disease compared to that of patients in the rest of the world. Observational data reported to the Fabry Registry were obtained from untreated patients or prior to treatment with enzyme replacement therapy. As of October 1, 2010, 3,752 patients were enrolled in the Fabry Registry worldwide, including 333 patients within Latin America. Latin American patients tended to be younger than Fabry Registry patients enrolled in the rest of the world: mean current age 35.5 years versus 39.2 years for men ( $p<0.05$ by $t$-test), mean age 37.8 years versus 43.6 years for women $(p<0.05$ by $t$-test). A smaller percentage of Latin American patients have received enzyme replacement therapy, compared to patients in the rest of the world: $67 \%$ versus $80 \%$ for men, and $19 \%$ versus $39 \%$ of women, respectively. Thirty-one percent of men and $22 \%$ of women in Latin America reported experiencing a significant cardiovascular, renal, or cerebrovascular event, at a mean age of $35 \pm 12.6$ years in men and $44 \pm 12.3$ years in women. Cardiovascular events were the most common type of initial clinical event among men and women in Latin America. The medical community in Latin America should be aware of Fabry disease as a possible cause of renal or cardiac dysfunction. Increased awareness will facilitate prompt diagnosis and initiation of treatment.
\end{abstract}

\section{Background}

Fabry disease is a rare X-linked lysosomal storage disorder caused by decreased or absent activity of the enzyme $\alpha$-galactosidase A ( $\alpha$-Gal A) (Brady et al. 1967). This condition results in the accumulation of glycolipids - mainly globotriaosylceramide (GL-3) in lysosomes from endothelial 
cells, fibroblasts, pericytes from dermal tissue, heart, kidney, and the autonomic nervous system (Desnick et al. 2001; Kolodny and Pastores 2002). The global incidence of Fabry disease is has been estimated as 1:40,000 males (Desnick et al. 2001) and as 1:117,000 in the general population (Meikle et al. 1999). However, patients who have atypical or few symptoms may not be diagnosed with Fabry disease, which makes it difficult to determine a precise incidence or prevalance of the disease. . Furthermore, newborn screening studies have suggested that the prevalence of various types of mutations in the gene encoding $\alpha$-Gal A may actually be much higher (for review, see [Germain 2010]).

Because Fabry disease is X-linked, men usually experience more severe manifestations than women (Desnick et al. 2001). However, a significant portion of heterozygous women also develop symptoms (Wilcox et al. 2008). The first symptoms of Fabry disease often appear during childhood, including acroparesthesias, abdominal pain, diarrhea, hypohidrosis or anhidrosis, corneal verticillata, and angiokeratoma (Desnick et al. 2001; Hopkin et al. 2008; Nguyen et al. 2005). The most serious complications of Fabry disease, including renal failure, cardiovascular events, and stroke usually manifest during adulthood (Desnick et al. 2001; Germain 2010; Wilcox et al. 2008). The increased availability of renal replacement therapy over the past several decades has extended the lifespan of Fabry patients (Branton et al. 2002; Mehta et al. 2009; Waldek et al. 2010). Cardiovascular disease is now the most commonly reported cause of death among both men and women with Fabry disease (Mehta et al. 2009; Waldek et al. 2010). The life expectancy (calculated from birth) for patients with Fabry disease is 58.2 years for men and 75.4 years for women, respectively (Waldeck et al. 2009).

The clinical diagnosis of Fabry disease can be difficult, because its signs and symptoms are often mistaken for other, more common diseases. In males, the demonstration of deficient enzymatic activity of $\alpha$-galactosidase $A$ in plasma or leukocytes can be used to confirm diagnosis (Brady et al. 1967). Enzyme activity can also be analyzed in dried blood spots on filter paper (Chamoles et al. 2001), which facilitates the screening of large populations. However, positive results obtained by this method should be corroborated by analysis of enzyme activity in plasma or leukocytes. Measurement of enzymatic activity is less useful in female heterozygotes, who often have $\alpha$-galactosidase A activity levels within the normal range. Genotyping should be used for diagnostic confirmation in heterozygous females (Linthorst et al. 2008). Furthermore, it is important that physicians obtain a complete medical history and perform a detailed pedigree analysis as the adverse consequences associated with delayed diagnosis include not only medical morbidity, but also psychological damage, loss of educational opportunities, and loss of economic productivity (Gold et al. 2002; Cole et al. 2007).

Springer
Currently, the only specific treatment for Fabry disease is enzyme replacement therapy (ERT) with recombinant human $\alpha$-Gal A (Eng et al. 2001; Schiffmann et al. 2001). Two forms of ERT are commercially available: agalsidase alfa (Shire Human Genetic Therapies, Inc. Cambridge, MA, USA) and agalsidase beta (Genzyme, a Sanofi company, Cambridge, MA, USA). The Fabry Registry is a global observational database that compiles clinical and laboratory data from patients with Fabry disease; it was established to further investigate the long-term effects of ERT and the natural progression of Fabry disease in a larger population. The purpose of these analyses was to compare key demographic and clinical characteristics of untreated Fabry Registry patients in Latin America (LATAM) to that of untreated patients in the rest of the world (ROW).

\section{Methods}

All patients with Fabry disease are eligible to enroll in the Fabry Registry, regardless of age, gender, symptoms, or whether or not they are receiving ERT from any commercial source. Patient and physician participation is voluntary and all patients sign informed consent forms that have been reviewed and approved by local Institutional Review Boards/ Ethics Committees. The Fabry Registry provides participating physicians with a recommended schedule of key clinical and laboratory assessments (http://www.fabryregistry.com). However, treating physicians determine the actual frequency of assessments according to a patient's individualized needs.

Fabry Registry patients in LATAM included those in Argentina, Brazil, Chile, Colombia, Mexico, Peru, Uruguay, and Venezuela. Patients in the ROW included all Fabry Registry patients except those in LATAM.

These analyses include data reported to the Fabry Registry as of October 1, 2010. Clinical parameters included in the analysis were chronic kidney disease (CKD) stage, urine protein to creatinine ratio, and left ventricular posterior wall thickness. Data were collected from treatment-naïve patients or, if a patient was treated, baseline data were analyzed (i.e., prior to the initiation of ERT). Patients whose ERT status was not reported to the Fabry Registry ( 7 men and 1 woman in LATAM) were not included in the clinical event analyses. Clinical parameter data were ascertained prior to and up to 1 month after the first agalsidase beta infusion, among adults who ever received agalsidase beta as a source of ERT $(\geq 18$ years old at the time of first infusion).

Clinical events that occurred prior to the initiation of ERT or in untreated patients were included in these analyses. Cardiovascular clinical events were defined as myocardial infarction, cardiac syncope, congestive heart failure, angina pectoris, or significant cardiac procedures (e.g. pacemaker or other implantable cardiac device placement, bypass, stent 


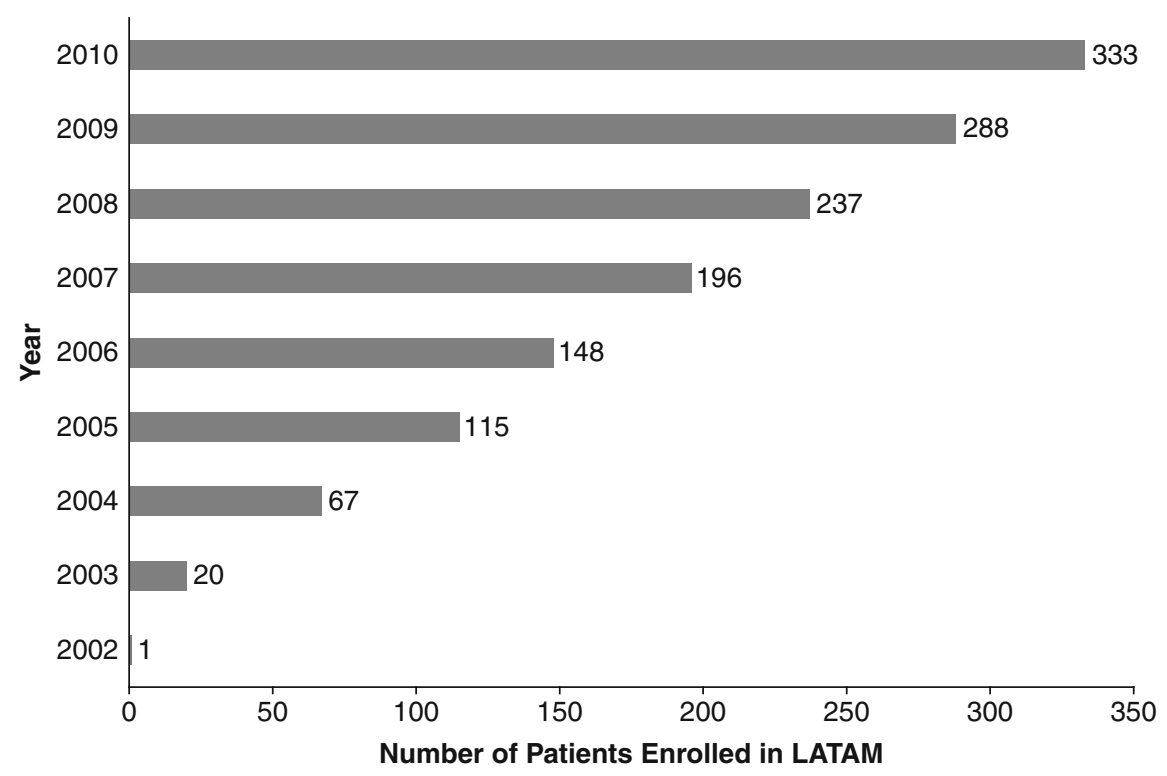

Fig. 1 Enrollment in the Fabry Registry within LATAM by year. Data shown represent cumulative enrollment of LATAM patients in the Fabry Registry as of December 31 of each year except 2010, which shows enrollment as of October 1, 2010

placement, valve replacement, transplantation), or arrhythmias. Arrhythmias may have been reported to the Fabry Registry as cardiovascular events, or detected by ECG in a follow-up visit or reported in a patient's medical history. Cerebrovascular events were defined as stroke. Renal events were defined as receiving chronic dialysis (40 days or longer) or kidney transplantation. Estimated glomerular filtration rate (eGFR) was calculated in $\mathrm{mL} / \mathrm{min} / 1.73 \mathrm{~m}^{2}$ from serum creatinine values, using the Chronic Kidney Disease Epidemiology Collaboration (CKD-EPI) equation (Levey et al. 2009).

To better understand the population of patients from LATAM, demographic characteristics of the patients enrolled in LATAM were compared to that of the patients enrolled in the Fabry Registry in the ROW. Tests of the null hypothesis of equal distributions between the cohorts were conducted by the $t$-test. Statistical analyses were performed using SAS statistical software version 9.1 (SAS Institute Inc., Cary, NC). Demographics and clinical characteristics of the cohorts are presented using summary statistics. An alphalevel of 0.05 was used as the cut-point to determine statistical significance.

\section{Results}

\section{Enrollment}

As of October 1, 2010, 333 patients were enrolled in the Fabry Registry within LATAM and 3,419 patients were enrolled in the ROW. Enrollment in the Fabry Registry in
LATAM has steadily increased since the first patient was enrolled in this region in 2002 (Fig. 1).

\section{Demographics}

Table 1 shows that patients in LATAM were significantly younger than patients enrolled in the ROW. As of October 1,2010 , the mean age of men in LATAM was 35.5 years versus 39.2 years for men in the ROW $(p=0.0054)$; the mean age of women in LATAM was 37.8 years versus 43.6 years for women in the ROW $(p<0.0001)$. Thirteen percent of Fabry Registry patients enrolled in LATAM were children ( $<18$ years old as of October 1, 2010), compared to $11 \%$ of patients in the ROW. The mean age at the time Fabry disease was diagnosed was similar among patients in LATAM and patients in the ROW by gender, but men tended to be diagnosed at a younger age than women in both populations (Table 1).

\section{ERT Treatment Status}

A substantially smaller percentage of women in LATAM have ever received ERT, compared to LATAM men (19\% versus 67\%), as shown in Fig. 2. Among both genders, patients in LATAM were less likely to receive ERT than patients in the ROW. Seventy percent of LATAM men have received ERT, compared to $80 \%$ of men in the ROW ( $p=0.0008) ; 19 \%$ of LATAM women have received ERT, compared to $39 \%$ of women in the ROW ( $<<0.0001$ ) (Fig. 2). 
Table 1 Summary of demographic characteristics of Fabry registry patients in LATAM and the ROW

\begin{tabular}{|c|c|c|c|c|}
\hline & \multicolumn{2}{|l|}{ LATAM } & \multicolumn{2}{|l|}{ ROW } \\
\hline & Males & Females & Males & Females \\
\hline Patients in the Registry, $n$ & 166 & 167 & 1679 & 1740 \\
\hline Current Age (years), $n$ & 166 & 167 & 1678 & 1739 \\
\hline Mean $\pm \mathrm{SD}$ & $35.5 \pm 13.71^{\mathrm{a}}$ & $37.8 \pm 17.81^{\mathrm{b}}$ & $39.2 \pm 16.81$ & $43.6 \pm 17.87$ \\
\hline Median (min, max) & $34.4(5,78)$ & $36.2(5,85)$ & $41.4(1,85)$ & $44.5(1,90)$ \\
\hline \multicolumn{5}{|l|}{ Age distribution, $n(\%)$} \\
\hline Pediatric $(<18$ years $)$ & $17(10.2)$ & $27(16.2)$ & $210(12.5)$ & $151(8.7)$ \\
\hline Adult ( $\geq 18$ years $)$ & $149(89.8)$ & $140(83.8)$ & $1,468(87.4)$ & $1,588(91.3)$ \\
\hline Unknown & 0 & 0 & $1(0.1)$ & $1(0.1)$ \\
\hline Age at diagnosis of FD (years), $n$ & 161 & 160 & 1,662 & 1676 \\
\hline Mean $\pm \mathrm{SD}$ & $28.6 \pm 13.35$ & $32.3 \pm 17.15$ & $27.3 \pm 17.28$ & $33.6 \pm 18.16$ \\
\hline Median $(\min , \max )^{\mathrm{c}}$ & $28.3(2,62)$ & $30.7(0,77)$ & $25.3(0,81)$ & $32.8(0,82)$ \\
\hline
\end{tabular}

Data shown represent that reported to the Fabry Registry as of October 1, 2010.

${ }^{\mathrm{a}} p=0.0054$ for men in LATAM versus men in the ROW, by $t$-test

${ }^{\mathrm{b}} p<0.0001$ for women in LATAM versus women in the ROW, by $t$-test

${ }^{\mathrm{c}}$ Patients with a prenatal age at diagnosis were considered to have an age at diagnosis of zero.

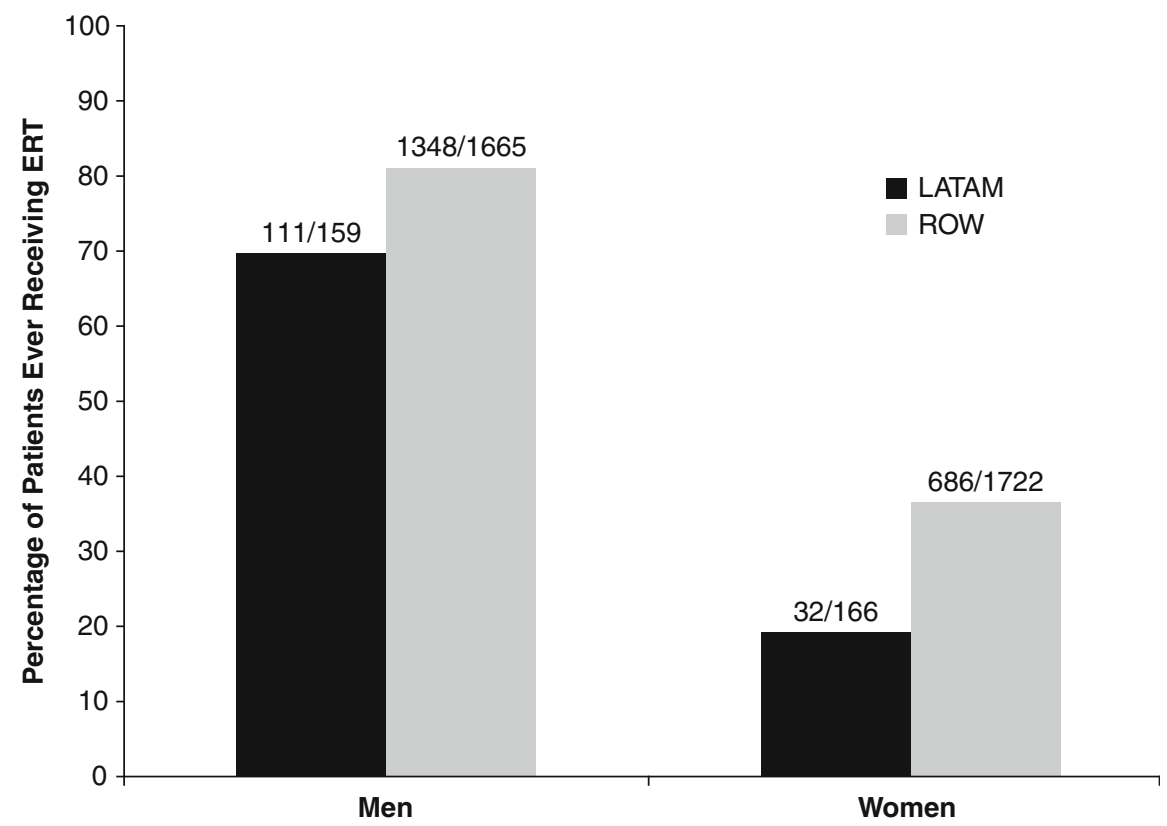

Fig. 2 ERT treatment status among men and women enrolled in the Fabry Registry in LATAM and in the ROW. Percentages were calculated based on the number of patients who reported ever receiving ERT from any source, excluding patients whose ERT status was not

\section{Clinical Events}

Clinical events were evaluated in the 325 patients from LATAM who had a known ERT status. Thirty-one percent of men (50 of 159) and $22 \%$ of women (37 of 166) in LATAM reported experiencing a significant cardiovascu- reported to the Fabry Registry. The numbers above each bar represent the number of patients who had reported receiving ERT/total number of patients in each category. For men, LATAM vs ROW $p=0.0008$; for women, LATAM vs ROW $p<0.0001$, by Chi-square test

lar, renal, or cerebrovascular event, as defined in Methods. The mean age at the time of the first clinical event was $35 \pm 12.6$ years in men and $44 \pm 12.3$ years in women. Cardiovascular events were the most common type of initial clinical event among men and women in LATAM. 
Table 2 Summary of clinical events and age at first clinical event

\begin{tabular}{llll}
\hline & & Males & Females \\
\hline Number of patients & & 159 & 166 \\
Patients reporting any type of clinical event & $n(\%)$ & $50(31.4)$ & $37(22.3)$ \\
Age at first event (years) & Mean (SD) & $35.4(12.58)$ & $36.3(0,66)$ \\
& Median (min, max) & $30(18.9)$ & $44.3(12.31)$ \\
Patients reporting cardiovascular event & $n(\%)$ & $37.8(15.19)$ & $47.2(13,71)$ \\
Age at first cardiovascular event (years) & Mean (SD) & $42.5(0,73)$ & $44(20.5)$ \\
& Median (min, max) & $24(15.1)$ & $45.5(12.50)$ \\
Patients reporting renal event & $n(\%)$ & $35.6(10.93)$ & $47.6(13,71)$ \\
Age at first renal event (years) & Mean (SD) & $35.9(19,66)$ & $4(2.4)$ \\
& Median (min, max) & $41.4(6.84)$ \\
Patients reporting stroke event & $n(\%)$ & $41.2(14.40)$ & $39.6(36,51)$ \\
Age at first stroke event (years) & Mean (SD) & $39.6(24,74)$ & $40.1(8.42)$
\end{tabular}

Data are from LATAM patients who had reported whether or not they had ever received ERT. All data were collected prior to initiation of ERT or are from patients who had not received ERT. Patients whose ERT status was unknown (7 men and 1 woman) were excluded from these analyses. Data shown represent that reported to the Fabry Registry as of October 1, 2010

Cardiovascular Manifestations

As shown in Table $2,19 \%$ of men (30 of 159$)$ and $21 \%$ of women (34 of 166) in LATAM reported a cardiovascular event, at a mean age of 38 and 46 years, respectively. The most common type of cardiovascular event was arrhythmia (any type), which was reported by $16 \%$ of men (26 of 159) and $19 \%$ of women (32 of 166). Arrhythmias included those reported to the Fabry Registry as cardiovascular events as well as those detected during ECG exams or reported in a patient's medical history. Less common cardiovascular events included significant cardiac procedures ( 8 of 159 men [5\%] and 2 of 166 women [1\%]), myocardial infarction (5 of 159 men [3\%] and no women), angina pectoris (4 of 159 men [3\%] and 3 of 166 women [2\%]), and heart failure ( 1 of 159 men [0.6\%] and 1 of 166 women [0.6\%]). Echocardiographic exam data were reported for 163 patients in LATAM. Among these, 18\% percent of men (29 of 72) and 15\% of women (25 of 91) exhibited left ventricular hypertrophy (LVH).

\section{Renal Manifestations}

Fifteen percent of men in LATAM (24 of 159) had a renal event (kidney transplant or chronic dialysis), at a mean age of $36 \pm 10.9$ years (Table 2). Two percent of women in LATAM (4 of 166) had a renal event, at a mean age of $41 \pm 6.8$ years. Among these patients, 10 of the 24 men and 2 of the 4 women had kidney transplants.
Cerebrovascular Manifestations

Five percent of men ( 8 of 159) and $2 \%$ of women ( 3 of $166)$ in LATAM reported having a stroke, at a mean age of $41 \pm 14.4$ years and $40 \pm 8.4$ years, respectively. One percent of men ( 2 of 159 ) and $5 \%$ of women ( 8 of 166) reported a transient ischemic attack (TIA).

\section{Pre-Treatment Clinical Characteristics}

Among the adult Fabry Registry patients in LATAM who reported receiving agalsidase beta treatment, 115 had reported some type of baseline echocardiographic and/or renal data to the Fabry Registry. Prior to beginning agalsidase beta treatment, among patients with available data, $38 \%$ of men (15 of 40 ) and $35 \%$ of women (7 of 20 ) exhibited left ventricular hypertrophy (Table 3). This is lower than the percentage of adults in the rest of the world who had left ventricular hypertrophy prior to beginning agalsidase beta treatment $(57 \%$ of men and $48 \%$ of women, Table 3$)$. Baseline renal function data (CKD stage as determined by eGFR) were available for 84 adult patients who later received ERT with agalsidase beta (Table 3). Prior to beginning treatment, 19 of 62 adult men in LATAM (31\%) were in CKD Stages $3-5\left(\right.$ eGFR $\left.<60 \mathrm{~mL} / \mathrm{min} / 1.73 \mathrm{~m}^{2}\right)$. Four of 22 adult Latin American females (18\%) were in CKD Stages 3-5 prior to beginning treatment. These are similar to the percentages of men and women in the ROW 
Table 3 Summary of pre-treatment renal and cardiac characteristics of adult Fabry registry patients in LATAM and the ROW

\begin{tabular}{|c|c|c|c|c|}
\hline & \multicolumn{2}{|c|}{ Adults in Latin America (LATAM) } & \multicolumn{2}{|c|}{ Adults inrest of world } \\
\hline & Males & Females & Males & Females \\
\hline Adult patients who ever received agalsidase beta, $N$ & 86 & 29 & 1001 & 511 \\
\hline Pre-treatment left posterior wall thickness (LPWT), $n(\%)$ & 40 & 20 & 572 & 343 \\
\hline LPWT $<12 \mathrm{~mm}$ & $25(62.5)$ & $13(65.0)$ & $248(43.4)$ & $178(51.9)$ \\
\hline LPWT $\geq 12 \mathrm{~mm}$, indicating left ventricular hypertrophy (LVH) & $15(37.5)$ & $7(35.0)$ & $324(56.6)$ & $165(48.1)$ \\
\hline \multicolumn{5}{|l|}{ Age at first report of LVH (years) } \\
\hline Mean (SD) & $34.5(11.94)$ & $44.8(12.49)$ & $39.4(11.5)$ & $46.5(12.2)$ \\
\hline Median (min, max) & $32.7(20,69)$ & $47.9(21,72)$ & $39.2(18,81)$ & $47.3(18,76)$ \\
\hline Pre-Treatment CKD Stage, n (\%) & 62 & 22 & 840 & 440 \\
\hline Stage $1\left(\geq 90 \mathrm{~mL} / \mathrm{min} / 1.73 \mathrm{~m}^{2}\right)$ & $32(51.6)$ & $10(45.5)$ & $383(45.6)$ & $210(47.7)$ \\
\hline Stage $2\left(\geq 60-<90 \mathrm{~mL} / \mathrm{min} / 1.73 \mathrm{~m}^{2}\right)$ & $11(17.7)$ & $8(36.4)$ & $187(22.3)$ & $153(34.8)$ \\
\hline Stage $3\left(\geq 30-<60 \mathrm{~mL} / \mathrm{min} / 1.73 \mathrm{~m}^{2}\right)$ & $8(12.9)$ & $3(13.6)$ & $147(17.5)$ & $56(12.7)$ \\
\hline Stage $4\left(\geq 15-<30 \mathrm{~mL} / \mathrm{min} / 1.73 \mathrm{~m}^{2}\right)$ & $4(6.5)$ & $1(4.5)$ & $55(6.5)$ & $11(2.5)$ \\
\hline Stage $5\left(<15 \mathrm{~mL} / \mathrm{min} / 1.73 \mathrm{~m}^{2}\right)$ & $7(11.3)$ & 0 & $68(8.1)$ & $10(2.3)$ \\
\hline Pre-treatment urine protein to creatinine ratio, $n(\%)$ & 21 & 9 & 452 & 280 \\
\hline$<0.3 \mathrm{~g} / \mathrm{g}$ & $6(28.6)$ & $5(55.6)$ & 157 (34.7) & $120(42.9)$ \\
\hline$\geq 0.3-<1 \mathrm{~g} / \mathrm{g}$ & $6(28.6)$ & $2(22.2)$ & $113(25.0)$ & $63(22.5)$ \\
\hline$\geq 1-<3 \mathrm{~g} / \mathrm{g}$ & $4(19.0)$ & 0 & $129(28.5)$ & $62(22.1)$ \\
\hline$\geq 3 \mathrm{~g} / \mathrm{g}$ & $5(23.8)$ & $2(22.2)$ & $53(11.7)$ & $35(12.5)$ \\
\hline
\end{tabular}

Data are from adults who ever received agalsidase beta ( $\geq 18$ years old at the time of the first infusion) and reflect data available as of 01 October 2010. Data obtained prior to and up to 1 month after the first agalsidase beta infusion were included in these calculations. eGFR (estimated glomerular filtration rate) was calculated in $\mathrm{mL} / \mathrm{min} / 1.73 \mathrm{~m}^{2}$ from serum creatinine values, using the Chronic Kidney Disease Epidemiology Collaboration (CKD-EPI) equation

who were in CKD Stages 3-5 before beginning agalsidase beta treatment (Table 3). Relatively few patients in LATAM had pre-treatment urinary protein data reported to the Fabry Registry; 9 of 21 men and 2 of 9 women reported a urinary protein to creatinine ratio $\geq 1 \mathrm{~g} / \mathrm{g}$ prior to beginning agalsidase beta treatment.

\section{Discussion}

As the largest registry that tracks clinical data for patients with Fabry disease, the Fabry Registry is a unique source of valuable information about this disorder. The objective of these analyses was to better characterize the growing population of Fabry Registry patients in LATAM.

Enrollment in LATAM has steadily increased over the past 8 years, with 333 patients enrolled in LATAM as of October 1, 2010. Compared to Fabry Registry patients in the ROW, a higher percentage of Fabry Registry patients in LATAM are children and the overall mean current age is younger in LATAM than in the ROW. It is possible that larger average family size in LATAM compared to the ROW could contribute to this difference, as diagnosis of one family member could lead to the screening of a higher number of younger family members. However, the younger current age in LATAM versus the ROW was not accompanied by a corresponding difference in age at diagnosis. It is not clear why a statistically significant difference was not observed in age at diagnosis, but there is a great deal of variation and a large standard deviation in the reported age at diagnosis. In any case, the high percentage of young patients enrolled in the Registry is very encouraging, as it suggests that physicians in LATAM are diagnosing Fabry disease in young patients. However, it is also important to regularly monitor these young patients; children in LATAM tend to have clinical data reported to the Fabry Registry less frequently than adults (Fabry Registry database).

As in the ROW, women in LATAM were less likely to receive ERT than men. Although some women have relatively mild symptoms of Fabry disease, others experience substantial manifestations of Fabry disease, including serious clinical events (Wilcox et al. 2008). Within LATAM, 37 of 166 women had a clinical event during the natural history period (i.e., prior to initiating any ERT). Overall, Fabry Registry patients in LATAM were less likely to have received ERT than patients in the ROW. This may be related to the fact that ERT became widely commercially available earlier in many countries in the ROW than in LATAM and may also be a reflection of differences in access to healthcare. 
Overall, $31 \%$ of men and $22 \%$ of women in LATAM experienced a clinical event during the natural history period, which is similar to what has been reported for the global Fabry Registry population (31\% of men and $20 \%$ of women) (Wilcox et al. 2008). The most common initial events reported by both genders were cardiovascular events, which is also similar to what has been reported for the overall Fabry Registry population (Wilcox et al. 2008). A retrospective chart review of 447 untreated patients also reported that cardiovascular events were the most common type of event in both genders (Schiffmann et al. 2009). The most common type of cardiovascular event reported was arrhythmia; overall, $18 \%$ of patients in LATAM reported some type of cardiac arrhythmia. This is consistent with other reports describing arrhythmias as the most common and earliest type of cardiac manifestation of Fabry disease (Linhart et al. 2007; Schiffmann et al. 2009; Wilcox et al. 2008). Among the LATAM patients who received agalsidase beta and for whom baseline echocardiographic data were reported, a smaller percentage displayed left ventricular hypertrophy, compared to patients in the ROW. This may be related to the fact that the patients in LATAM were younger than patients in the ROW.

Fifteen percent of men and $2 \%$ of women in LATAM required renal replacement therapy, through either dialysis or a kidney transplant, at a mean age of 36 years for men and 41 years for women. This is remarkably similar to what has been reported for the overall Fabry Registry population. Ortiz et al. reported that $14 \%$ of untreated men and $2 \%$ of untreated women in the global Fabry Registry required dialysis or a kidney transplant at a men age of 39 years (men) and 40 years (women) (Ortiz et al. 2010). Unfortunately, relatively few patients in LATAM reported detailed renal clinical data to the Fabry Registry, in terms of eGFR and/or urinary protein levels. The data that are available suggest that similar percentages of patients in LATAM and in the ROW exhibit CKD Stage 3 or higher prior to initiating ERT (approximately 30\%).

A small percentage of patients in LATAM reported a stroke: $5 \%$ of men and $2 \%$ of women at a mean age of 41 years and 40 years, respectively. This is generally consistent or slightly lower than what has been reported for the overall Fabry Registry population; Sims et al. reported that $7 \%$ of untreated men and $4 \%$ of untreated women experienced a stroke (Sims et al. 2009). In the global Fabry Registry population, the age at first stroke was 40 for men and 46 for women (Sims et al. 2009).

Many of the manifestations of Fabry disease resemble other, more common disorders. Consequently, patients with Fabry disease are frequently not diagnosed until long after the onset of symptoms. In 2007, the average time from symptom onset to diagnosis among Fabry Registry patients was 14 years for men and 16 years for women (Wilcox et al. 2008). This is concerning, because Fabry disease is treatable and there is considerable evidence that ERT is most effective when it is initiated early, before the onset of substantial organ damage (Banikazemi et al. 2007; Germain et al. 2007; Warnock et al. 2011). Increased awareness of Fabry disease among the general medical community will facilitate prompt diagnosis and reduce delays in treatment, both in LATAM and in the ROW. In addition, physicians should be encouraged to obtain a thorough medical history for all patients and to screen potentially affected family members.

Various limitations are associated with analyzing observational data from a disease registry, particularly for rare diseases with small numbers of patients. For example, not all patients have reported all types of clinical data to the Fabry Registry. While a schedule of recommended clinical assessments is provided, treating physicians determine the actual type and frequency of assessments according to individual patients' needs. In addition, some patients report data to the Fabry Outcome Survey, a smaller registry that tracks patients with Fabry disease; the baseline clinical characteristics of patients enrolled in the two registries may differ.

In summary, enrollment in the Fabry Registry within LATAM has increased substantially over the past several years, which may indicate increased awareness of Fabry disease in the LATAM region and increased interest in contributing data to this important database. Over time, as the Fabry Registry continues to collect clinical data from patients in LATAM, we will gain a better understanding of the nature of Fabry disease and treatment outcomes in this patient population.

Aknowledgements The authors would like to thank the patients who have agreed to participate in the Fabry Registry as well as the physicians and research coordinators that have entered clinical data on these patients. We also acknowledge our colleagues at Genzyme (Cambridge, MA): Dana Beitner-Johnson $\mathrm{PhD}$, for revising the paper for clarity in English and Badari Gudivada, for statistical programming support.

\section{Synopsis}

The Fabry Registry has collected data from 333 Latin American patients with Fabry disease; demographic and clinical characteristics of these patients were compared to Fabry Registry patients in the rest of the world. 


\section{Author Contributions}

\begin{tabular}{|c|c|c|c|c|c|}
\hline Name & Design & Analysis and data interpretation & Write the article & Content Critical review & Final approval \\
\hline J Villalobos & $\mathrm{X}$ & $\mathrm{X}$ & $\mathrm{X}$ & $\mathrm{X}$ & $\mathrm{X}$ \\
\hline JM Politei & & & & $\mathrm{X}$ & $\mathrm{X}$ \\
\hline AM Martins & & & & $\mathrm{X}$ & $\mathrm{X}$ \\
\hline G Cabrera & & & & $\mathrm{X}$ & $\mathrm{X}$ \\
\hline H Amartino & & & & $\mathrm{X}$ & $\mathrm{X}$ \\
\hline R Lemay & $\mathrm{X}$ & $\mathrm{X}$ & & $\mathrm{X}$ & $\mathrm{X}$ \\
\hline S Ospina & & $\mathrm{X}$ & & $\mathrm{X}$ & $\mathrm{X}$ \\
\hline S Suarez Ordoñez & & & $\mathrm{X}$ & $\mathrm{X}$ & $\mathrm{X}$ \\
\hline C Varas & & & & $\mathrm{X}$ & $\mathrm{X}$ \\
\hline
\end{tabular}

\section{Author (Guarantor for the Article)}

Jacobo Villalobos, M.D.

\section{Competing Interest Statement}

The authors declare independence from the sponsors and declare that the content of the article has not been influenced by the sponsors.

\section{Funding}

Genzyme sponsors the Fabry Registry.

\section{Ethics Approval}

The Fabry Registry Program has the approval of ethics committees. A specific ethics approval for this paper was not required and these documents are available upon request.

\section{Conflict of Interest Disclosures}

JV, JMP, AMM, SO, GC, and CV are members of the Fabry Registry Board of Advisors. JV, JMP, AMM, and GC have received research funds or travel support from Genzyme as well as speaking fees from Genzyme. HA has received speaking fees from Genzyme. RL is a full-time employee of Genzyme and SSO works as a contractor for Genzyme.

\section{References}

Banikazemi M, Bultas J, Waldek S et al (2007) Agalsidase-beta therapy for advanced Fabry disease: a randomized trial. Ann Intern Med 146:77-86

Brady RO, Gal AE, Bradley RM, Martensson E, Warshaw AL, Laster L (1967) Enzymatic defect in Fabry's disease. Ceramidetrihexosidase deficiency. N Engl J Med 276:1163-1167
Branton MH, Schiffmann R, Sabnis SG et al (2002) Natural history of Fabry renal disease: influence of alpha-galactosidase A activity and genetic mutations on clinical course. Medicine (Baltimore) $81: 122-138$

Chamoles NA, Blanco M, Gaggioli D (2001) Fabry disease: enzymatic diagnosis in dried blood spots on filter paper. Clin Chim Acta 308:195-196

Cole AL, Lee PJ, Hughes DA et al (2007) Depression in adults with Fabry disease: a common and under-diagnosed problem. J Inherit Metab Dis 30:943-951

Desnick RJ, Ioannou YA, Eng CM (2001) Alpha-Galactosidase A deficiency: Fabry disease. In: Scriver C, Beaudet A (eds) The metabolic bases of inherited disease. McGraw-Hill, New York, pp 3733-3774

Eng CM, Guffon N, Wilcox WR et al (2001) Safety and efficacy of recombinant human alpha-galactosidase A-replacement therapy in Fabry's disease. N Engl J Med 345:9-16

Germain DP, Waldek S, Banikazemi M et al (2007) Sustained, longterm renal stabilization after 54 months of agalsidase beta therapy in patients with Fabry disease. J Am Soc Nephrol 18:1547-1557

Germain DP (2010) Fabry disease. Orphanet J Rare Dis 5:30

Gold KF, Pastores GM, Botteman MF et al (2002) Quality of life of patients with Fabry disease. Qual Life Res 11:317-327

Hopkin RJ, Bissler J, Banikazemi M et al (2008) Characterization of Fabry disease in 352 pediatric patients in the Fabry Registry. Pediatr Res 64:550-555

Kolodny EH, Pastores GM (2002) Anderson-Fabry disease: extrarenal, neurologic manifestations. J Am Soc Nephrol 13(Suppl 2): S150-153

Levey AS, Stevens LA, Schmid CH et al (2009) A new equation to estimate glomerular filtration rate. Ann Intern Med 150:604-612

Linhart A, Kampmann C, Zamorano JL et al (2007) Cardiac manifestations of Anderson-Fabry disease: results from the international Fabry outcome survey. Eur Heart J 28:1228-1235

Linthorst GE, Poorthuis BJ, Hollak CE (2008) Enzyme activity for determination of presence of Fabry disease in women results in $40 \%$ false-negative results. J Am Coll Cardiol 51:2082, Author reply $82-83$

Mehta A, Clarke JT, Giugliani R et al (2009) Natural course of Fabry disease: changing pattern of causes of death in FOS - Fabry Outcome Survey. J Med Genet 46:548-552

Meikle PJ, Hopwood JJ, Clague AE, Carey WF (1999) Prevalence of lysosomal storage disorders. JAMA 281:249-254 
Nguyen TT, Gin T, Nicholls K, Low M, Galanos J, Crawford A (2005) Ophthalmological manifestations of Fabry disease: a survey of patients at the Royal Melbourne Fabry Disease Treatment Centre. Clin Experiment Ophthalmol 33:164-168

Ortiz A, Cianciaruso B, Cizmarik M et al (2010) End-stage renal disease in patients with Fabry disease: natural history data from the Fabry Registry. Nephrol Dial Transplant 25:769-775

Schiffmann R, Kopp JB, Austin HA 3rd et al (2001) Enzyme replacement therapy in Fabry disease: a randomized controlled trial. JAMA 285:2743-2749

Schiffmann R, Warnock DG, Banikazemi M et al (2009) Fabry disease: progression of nephropathy, and prevalence of cardiac and cerebrovascular events before enzyme replacement therapy. Nephrol Dial Transplant 24:2102-2111

Sims K, Politei J, Banikazemi M, Lee P (2009) Stroke in Fabry disease frequently occurs before diagnosis and in the absence of other clinical events: natural history data from the Fabry Registry. Stroke 40:788-794

Waldeck S, Patel MR, Banikazemi M, Lemay R, Lee P (2009) Life expectancy and cause of death in males and females with Fabry disease: findings from the Fabry Registry. Genet Med 11 (11):790-796

Waldek S, Germain DP, Wanner C, Warnock DG (2010) Enzyme replacement therapy for Fabry's disease. Lancet 375:1523, Author reply $23-24$

Warnock DG, Ortiz A, Mauer M et al (2011) Renal outcomes of agalsidase beta treatment for Fabry disease: role of proteinuria and timing of treatment initiation. Nephrol Dial Transplant. doi:10.1093/ndt/gfr420

Wilcox WR, Oliveira JP, Hopkin RJ (2008) Females with Fabry disease frequently have major organ involvement: Lessons from the Fabry Registry. Mol Genet Metab 93:112-128 